The archived file is not the final published version of the article.

F. Grimm, G. Reichling, R. Ewert, J. Dierke, B. Noll, M. Aigner, Stochastic and Direct Combustion Noise Simulation of a Gas Turbine Model Combustor, Acta Acustica United with Acustica 103 (2017) 262-275, doi 10.3813/AAA.919055

(C) (Year) S. Hirzel Verlag/European Acoustics Association

The definitive publisher-authenticated version is available online at http://www.ingentaconnect.com/content/dav/aaua

http://dx.doi.org/10.3813/AAA.919055

Readers must contact the publisher for reprint or permission to use the material in any form 


\title{
Stochastic and Direct Combustion Noise Simulation of a Gas Turbine Model Combustor
}

\author{
Felix Grimm ${ }^{1)}$, Gilles Reichling ${ }^{1)}$, Roland Ewert ${ }^{2)}$, Juergen Dierke ${ }^{2)}$, \\ Berthold Noll ${ }^{1)}$, Manfred Aigner ${ }^{1)}$ \\ 1) German Aerospace Center (DLR), Institute of Combustion Technology \\ Pfaffenwaldring 38-40, 70569 Stuttgart, Germany. felix.grimm@dlr.de \\ 2) German Aerospace Center (DLR), Institute of Aerodynamics and Flow Technology \\ Lilienthalplatz 7, 38108 Braunschweig, Germany
}

1

Summary RANS. (IPM).
Combustion noise of a gas turbine model combustor operating in partially premixed mode under atmospheric conditions is simulated with both, a hybrid stochastic approach and a direct, scale resolving method. Results from the hybrid ansatz are compared with experimental data and with results from incompressible and compressible CFD simulations.

The hybrid time-domain method 3D FRPM-CN consists of a stochastic sound source reconstruction algorithm, the Fast Random Particle Method (FRPM) and sound propagation by linearized Euler Equations. The method is herein evaluated for its capability of Combustion Noise $(\mathrm{CN})$ prediction. Monopole sound sources are reconstructed by using an estimation of turbulence statistics from reacting, steady-state CFD-

As a direct approach, a Compressible Projection Method (CPM) is applied. It is an extension of conventional pressure-based methods for the treatment of compressible flows. This solution strategy is implemented as a fractional step scheme in the DLR Finite Volume based research code THETA. CFD results of CPM and RANS are furthermore compared to results from a conventional incompressible projection method

First, steady state and unsteady CFD simulations of flow field and combustion of the model combustor are compared to experimental data. Two equation modeling for turbulence and global chemistry treatment for combustion are employed. Turbulence in unsteady computations is depicted with a scale adaptive simulation (SAS). In a second step, the hybrid acoustics simulation setup for the model combustor is introduced. Selected results are presented and 3D FRPMCN pressure spectra are compared to experimental data and results from CPM. Finally, computational turnaround times of hybrid and direct approach are evaluated and opposed.

\section{Introduction}

Noise emission has become an issue with high social, environmental and economic relevance throughout the last years, especially in the field of aviation. More and more strict regulations regarding aircraft engine noise emissions therefore ensure that the understanding of noise generation mechanisms and on top of that noise reduction measures remain a highly relevant research topic. However, substantial progress was made in terms of fan, turbine and jet noise reduction for example by increasing the overall engine bypass ratio. As a consequence, the relative contribution of combustion noise to the overall noise level increased. Therefore it is important to gain a detailed understanding of combustion noise generation mechanisms as a first step in order to be able to derive effective noise reduction techniques.

The phenomenon of broadband combustion noise due to the interaction of chemical processes with flow unsteadiness can be in principle modeled in two ways: The direct approach, meaning partially or fully scale resolving compressible DNS or LES calculations, which are often difficult to handle and computationally extremely expensive. As an alternative, there are the so called hybrid approaches, separating CFD (Computational Fluid Dynamics) and CCA (Computational Combustion Acoustics [6]) scales. They provide a large potential for computational savings and the possibility to apply specifically optimized methods to each part of the problem [5], since acoustic pressure fluctuations are usually in the order of magnitude of the CFD computational error.

A fairly popular approach in hybrid techniques in CCA is the limitation of scale resolving LES or DNS to an assumed sound source region, where several methods have been developed in especially the past twenty years with a variety of different source term formulations and models for sound propagation. Flemming et al. [18] for example made use of Lighthill's acoustic analogy. They formulated a source model based on the heat release expressed as density 
flucutations and Ihme et al. [23] identified the predominant combustion noise source as being linked to the chemical reaction rate in their progress variable combustion model. Both approaches focused on nonpremixed combustion. Zhang et al. [49] used a rearranged Lighthill wave equation with a heat release related source term provided from LES calculations. Brick et al. [4] employed an analytical Kirchhoff approach in order to predict far field radiation. Silva et al. [41] carried out both, direct scale resolving and hybrid simulations based on Phillips' analogy [36] and compared resulting pressure spectra for a swirl stabilized, premixed and confined flame. Their source mechanism was associated to the heat release rate. Another comparison between direct and hybrid combustion noise simulations was carried out by Zhang et al. [50], where open turbulent premixed and nonpremixed flames were treated. Their hybrid approach was based on Lighthill's equation and additionally, a spectral method was taken into consideration. Works based on spectral methods were also presented by Hirsch et al. [21] for the distribution of heat release in turbulent premixed combustion. Their model was utilized by Liu et al. [28] in a recent work on the prediction of combustion noise in an aero-engine combustor, with a similar scope as in the herein presented paper. Bui et al. [5] analyzed different source term components and identified the heat release as the predominant influence, while sound propagation was modeled with the so called Acoustic Perturbation Equations for Reacting Flows (APE-RF), thus a numerical approach for sound wave propagation. Their predominant source component was formulated as being proportional to density fluctuations $D \rho / D t$. A comprehensive discussion of combustion noise sources with provision of a detailed source term formulation was carried out by Candel et al. [6]. Similar to most of the previously quoted works, they identified a heat-release related monopole radiator as predominant source, especially in air-burning systems.

The hybrid method applied in this work is a timedomain approach, relying on stochastic sound source reconstruction, while sound propagation is computed with the linearized Euler equations. The in principle functioning of the method is depicted in Fig. 1.

Reacting CFD RANS simulations are carried out at first, delivering the mean flow, density and pressure field for sound propagation with the linearized basic equations and therefore a realistic depiction of refraction effects and sound spread. At the same time they provide source field one-point statistics in an assumed source region from the local turbulence quantities. Noise sources are reconstructed with FRPM, which are in this case temperature variance based and the noise sources are coupled to the LEEs as right hand side forcing. The basic equations together with the sound sources denote the overall acoustic model, and its every-timestep solution gives time-signals of

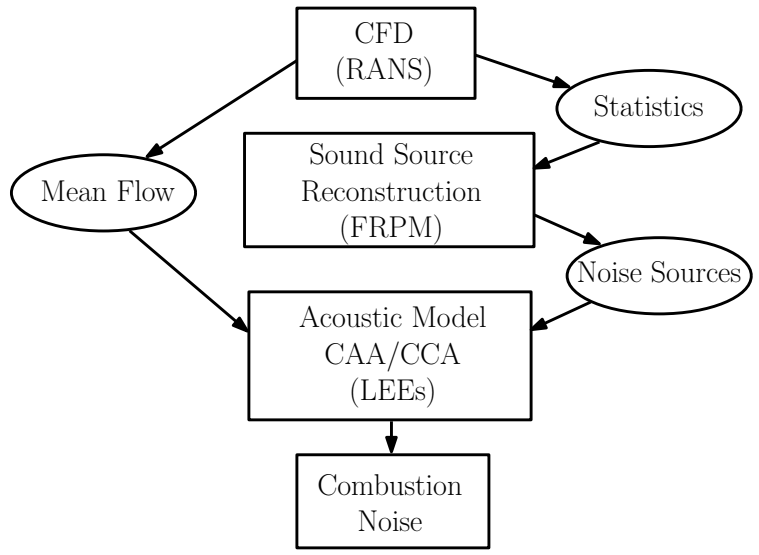

Figure 1: Scheme of the hybrid method FRPM-CN [20].

acoustic pressure fluctuations, which are transformed to sound pressure spectra at arbitrary observer positions.

The historical development of the herein presented stochastic ansatz was outlined by Mühlbauer et al. [33], starting with stochastic non-reacting approaches based on discrete Fourier modes in 1970 by Kraichnan [26] followed by several modified approaches in the 1990s [2,1] and 2000s [25,3], when the term of stochastic noise generation and radiation (SNGR) arose. The SNGR approach was based on synthesizing the turbulent velocity field with discrete Fourier modes, fed by RANS mean flow quantities and mainly applied to cold jets until then. The particular line of development for the stochastic, particle based hybrid ansatz with correlated sources which is pursued here started with the introduction of the RPM (Random Particle Mesh Method) by Ewert and Emunds [15]. Their RPM realized sources with spatio-temporal correlations based on local turbulence statistics for the applications mentioned previously.

The approach of combustion noise modeling utilized in this work was derived by Mühlbauer et al. [33], using the sound source reconstruction algorithm from Ewert [13], while the derivation of the source term formulation was inspired by the cold jet noise model of Tam and Auriault [44]. The physical source term model was derived from first principles, using a fundamental pressure-density relation, leading to the linearized Euler energy equation with a right hand side forcing [33], while the complete right hand side source formulation of the pressure-density relation was taken from Candel et al. [6]. The resulting formulation modeled with RPM was temperature variance based, while the variance field was determined by solving an additional transport equation according to Gerlinger [19] in the preceding CFD reacting RANS simulations. In a first approach to combustion noise prediction the RPM in conjunction with the acoustic perturbation equations (APEs) were used. The genuine APEs were 
introduced by Ewert and Schröder [17]. Later the source term model was reformulated on a more general basis, theoretically applicable to all reacting flow cases [33]. It was derived for the use in combination with the linearized Euler equations (LEE) and the approach was called RPM-CN (Random Particle Mesh Method for Combustion Noise Prediction) [33]. Mean flow field data and mean turbulence statistics for this causal approach were provided by steady-state RANS calculations, in view of potential computational savings compared to LES based methods.

On that basis, the method RPM-CN was advanced by Grimm et al. [20] by using the existing source term formulation but a different, highly efficient source reconstruction algorithm which is more suitable for technically relevant applications, the FRPM (Fast Random Particle Method) from Ewert et al. [14]. This approach, the so called FRPM-CN, was verified in terms of one- and two-point source statistics as well as far-field spectra reproduction ability [20]. In the presented work, a laboratory model combustor that features a broadband spectral combustion noise distribution is investigated with 3D FRPM-CN and a scale adaptive simulation approach in combination with a compressible projection method [39] and an incompressible approach for flow field and combustion evaluation are used. The hybrid approach 3D FRPM$\mathrm{CN}$ is validated in view of reproduction ability of absolute combustion noise levels for a complex, swirl stabilized test case with encasement. Furthermore, the performance of 3D FRPM-CN is compared to that of a direct method, not only for reproduction of combustion acoustics, but also regarding steady state CFD simulations as part of the process chain shown in Fig. 1. By elaborating and discussing the results of both, hybrid and direct methods, the potential of the presented hybrid, stochastic ansatz is shown, in particular with regard of computational turnaround times. The paper is organized as follows: First, the different theoretical aspects of the model are introduced and explained. After that, the CFD and CCA computational setups and specifications are shown and the respective results are compared to experimental data. In the same turn, the performance of the employed CFD-reacting RANS is evaluated by comparison with incompressible and compressible SAS simulations on profile lines of velocity components, temperature and temperature RMS. On that basis, selected results of 3D FRPM-CN are shown and reproduced combustion noise sound pressure spectra are compared to experiments and results from a scale-resolving compressible projection method. Finally, computational turnaround times of direct simulation and hybrid approach are opposed and discussed.

\section{Thermo-Fluiddynamics Framework}

First of all the theoretical framework is introduced by defining the basic equations of a reacting flow system, together with the formal description of the combustion model.

\subsection{Governing Equations}

The governing equations for a reacting flow in their compressible and conservative form, transport equations for mass, momentum, energy and species mass fractions, are

$$
\begin{gathered}
\frac{\partial \rho}{\partial t}+\nabla \cdot(\rho \boldsymbol{u})=0 \\
\frac{\partial(\rho \boldsymbol{u})}{\partial t}+\nabla \cdot(\rho \boldsymbol{u u})+\nabla p=\nabla \cdot \boldsymbol{\tau}_{\tau} \\
\frac{\partial(\rho h)}{\partial t}+\nabla \cdot(\rho \boldsymbol{u} h)-\frac{\partial p}{\partial t}-\boldsymbol{u} \cdot \nabla p=\nabla \cdot(\lambda \nabla T)+\boldsymbol{\tau}_{\tau}: \nabla \boldsymbol{u} \\
\frac{\partial\left(\rho Y_{\alpha}\right)}{\partial t}+\nabla \cdot\left(\rho \boldsymbol{u} Y_{\alpha}\right)=\nabla \cdot\left(\boldsymbol{D} \cdot \nabla Y_{\alpha}\right)+S_{\alpha}
\end{gathered}
$$

for $\alpha=1,2, \ldots, N_{s}-1$ species with mass fractions $Y_{\alpha}$ and the chemical source term $S_{\alpha}$ associated to each species. $\boldsymbol{\tau}_{\tau}$ and $\boldsymbol{D}$ are the stress and diffusion tensor, while $\lambda$ is the thermal conductivity. The term $\boldsymbol{\tau}_{\tau}: \nabla \boldsymbol{u}$ represents the rate of work for shape change in the case of a constant volume. The component $\nabla \cdot(\rho \boldsymbol{u u})$ in Eqn. (2) is defined as resulting in a column vector after application of the differential operator to the dyadic product $\rho \boldsymbol{u u}$. The same applies for the tensor of tensions in Eqn. (2), $\nabla \cdot \boldsymbol{\tau}_{\tau}$.

Pressure $p$ and density $\rho$ are inter-related by the thermal equation of state and the specific gas constant $R$ is expressed in terms of the component mass fractions $Y_{\alpha}$ and molar masses $M_{\alpha}$

$$
\rho=\frac{p_{r e f}+p}{R T} \quad \text { with } \quad R=\mathcal{R} \sum_{\alpha=1}^{N_{s}} \frac{Y_{\alpha}}{M_{\alpha}} .
$$

Equations (1) to (5) describe compressible reacting flow. The enthalpy is defined as

$$
h=\int_{T_{0}}^{T} c_{p} d T+\sum_{\alpha=1}^{N_{s}} \Delta h_{f, \alpha}^{0} Y_{\alpha},
$$

with the heat capacity $c_{p}$ and the standard formation 240 enthalpy $h_{f, \alpha}^{0}$ at reference conditions for species $\alpha$. ${ }^{241}$

\subsection{Combustion Modeling}

In the herein presented test-case, methane is burned with air under atmospheric conditions. Chemical reactions are modeled with a global reaction mechanism from Nicol et al. [35], originally containing five reaction steps. However it is used in a three-step form, 
since $\mathrm{NO}_{x}$ formation is not investigated. Methane and oxygen become monoxide and water, the formation of carbon dioxide takes place from carbon monoxide and oxygen, while the dissociation reaction is carbon dioxide to carbon monoxide and oxygen, reading

$$
\begin{aligned}
\mathrm{CH}_{4}+3 / 2 \mathrm{O}_{2} & \rightarrow \mathrm{CO}+2 \mathrm{H}_{2} \mathrm{O} \\
\mathrm{CO}+1 / 2 \mathrm{O}_{2} & \rightarrow \mathrm{CO}_{2} \\
\mathrm{CO}_{2} & \rightarrow \mathrm{CO}+1 / 2 \mathrm{O}_{2} .
\end{aligned}
$$

Global chemical reaction systems can be generally expressed as

$$
\sum_{\alpha=1}^{N_{s}} \nu_{\alpha, r}^{\prime} \mathrm{El}_{\alpha} \rightarrow \sum_{\alpha=1}^{N_{s}} \nu_{\alpha, r}^{\prime \prime} \mathrm{El}_{\alpha}
$$

$\nu_{\alpha, r}^{\prime}$ and $\nu_{\alpha, r}^{\prime \prime}$ are the stoichiometric coefficients of educts and products for a given species $\alpha$ and reaction $r$. Equations (7) to (9) can then be cast from Eqn. (10) by summarizing over all species $N_{s}$.

On the numerical simulation side chemistry is treated with the Eddy Dissipation Model (EDM) in conjunction with Finite Rate Chemistry (FRC) [29]. The herein investigated burner operates in partially premixed mode. The EDM alone significantly overpredicts chemical rates, since it is based on the assumption of the reaction being mixing-controlled and kinetics to happen infinitely fast. Therefore, overall reaction rates are corrected with Arrhenius based, kinetics controlled FRC-rates, evaluated from

$$
R R_{r}=\min \left(R R_{r}^{E D M}, R R_{r}^{F R C}\right),
$$

for a reaction $r$. Due to the use of this combined EDM/FRC model, partially premixed combustion is adequately depicted in the numerical simulation. The chemical source term in Eqn. (4) consequently is

$$
S_{\alpha}=M_{\alpha} \sum_{r=1}^{N_{r}}\left(\nu_{\alpha, r}^{\prime \prime}-\nu_{\alpha, r}^{\prime}\right) \cdot \min \left(R R_{r}^{E D M}, R R_{r}^{F R C}\right),
$$

with the molar masses $M_{\alpha}$ of species $\alpha$ by summarizing over all modeled reactions $N_{r}$. The reaction rates of EDM are

$$
\begin{aligned}
& R R_{r}^{E D M}= \\
& A \frac{\rho}{\tau_{T}}\left[\min \left(\min _{\alpha, \nu_{\alpha, r}^{\prime} \neq 0} \frac{Y_{\alpha}}{\nu_{\alpha, r}^{\prime} M_{\alpha}}, B \frac{\sum_{\alpha} Y_{\alpha}}{\sum_{\alpha} \nu_{\alpha, r}^{\prime \prime} M_{\alpha}}\right)\right],
\end{aligned}
$$

with the empirical constants $A=4$ and $B=0.5$. $Y_{\alpha}$ are mass fractions of species $\alpha . \quad \rho$ and $\tau_{T}$ denote density and local integral turbulent time-scale of the flow. As mentioned earlier, EDM reaction rates are assumed to be controlled by mixing processes of fuel and oxidizer due to turbulence, which implies that chemical processes are infinitely fast. Mixing controlled reaction is expressed in Eqn. (13), where $R R_{r}^{E D M} \sim 1 / \tau_{T}$. As a consequence, reaction rates can be significantly overpredicted, especially in the case of local non-equilibrium effects [24]. Therefore, overall rates are evaluated with Eqn. (11), which implies a correction with Arrhenius-function based FRC reaction rates. Those are evaluated from the products

$$
R R_{r}^{F R C}=k_{f, r} \prod_{\alpha=1}^{N_{s}} C_{\alpha}^{\nu_{\alpha, r}^{\prime}}-k_{b, r} \prod_{\alpha=1}^{N_{s}} C_{\alpha}^{\nu_{\alpha, r}^{\prime \prime}},
$$

with the concentrations $C_{\alpha}$ [40]. $k_{f, r}$ and $k_{b, r}$ are the forward and backward rate constants which are fitted to the prevailing thermodynamic conditions. They are modelled by assuming the following temperature dependency:

$$
k_{\zeta, r}=A_{r} T^{\beta_{r}} \exp \left(-\frac{E_{a, r}}{\mathcal{R} T}\right), \quad \text { for } \quad \zeta \in[f, b],
$$

where $E_{a, r}$ is the activation energy of reaction $r$ with the respective dimensionless temperature exponent $\beta_{r}$. $T$ represents temperature and $\mathcal{R}$ is the universal gas constant.

Since the employed combustion noise source term formulation is temperature variance based, an additional transport equation [19] for the temperature variance is solved. It reads

$$
\begin{gathered}
\bar{\rho} \nabla \cdot\left(\widetilde{T^{\prime \prime 2}} \boldsymbol{u}\right)-\nabla \cdot\left[\left(\frac{\mu}{P r}+\frac{\mu_{t}}{P r_{t}}\right) \nabla \widetilde{T^{\prime \prime 2}}\right] \\
=\underbrace{2 \frac{\mu_{t}}{P r_{t}}(\nabla \widetilde{T})^{2}}_{\text {production }} \underbrace{-\bar{\rho} C_{T} \frac{\widetilde{T^{\prime \prime 2}}}{\tau_{T}}}_{\text {dissipation }},
\end{gathered}
$$

with the model constant $C_{T}=2 . \mu$ and $\operatorname{Pr}$ are the viscosity and the Prandtl number. The indices $t$ and $T$ mean turbulent and turbulent temperature associated. The $\sim$ denotes Favré averaging. Equation (16) does not affect combustion modeling but is solved as a post-processing step based on the existing flow and combustion field solution.

\subsection{The Compressible Projection ${ }^{250}$ Method (CPM)

Results of combustion noise simulation is not only validated with experimental data, but also evaluated against a direct, compressible method. Therefore, the Compressible Projection Method from Reichling et al. [39] is taken into consideration. This approach extends the incompressible, pressure-based solver of the unstructured finite volume based CFD code THETA $[10,39]$ for the treatment of weakly compressible flows.

It was developed based on projection schemes from Chorin [7] and Temam [47]. The CPM iteratively solves Eqns. (1) to (5). First, the divergence constraint is computed at timestep $n$,

$$
\nabla \cdot \boldsymbol{u}_{n}=f\left(p_{n}, \nabla \boldsymbol{u}_{n}, \nabla p_{n}, \partial p_{n} / \partial t, \nabla T_{n}\right),
$$


as well as an interim solution $(*)$ of the velocity field $\boldsymbol{u}_{*}$,

$$
\left.\frac{\partial(\rho \boldsymbol{u})}{\partial t}\right|_{*}+\nabla \cdot\left[(\rho \boldsymbol{u})_{n} \otimes \boldsymbol{u}_{*}\right]+\nabla p_{n}=\nabla \boldsymbol{\tau}_{\tau, n},
$$

with $\otimes$ indicating the dyadic product. A pressure correction equation is then solved for $\delta p_{n+1}=p_{n+1}-$ $p_{n}$,

$$
\begin{aligned}
\Delta_{L} \delta p_{n+1} & -\frac{\alpha_{p r}}{f(\Delta t) \Delta t} \frac{\rho_{n}}{\gamma_{n}\left(p_{r e f}+p_{n}\right)} \delta p_{n+1} \\
& =f\left(\alpha_{p r}, \nabla \cdot\left(\rho_{n} \boldsymbol{u}_{*}\right), \boldsymbol{u}_{*}, \nabla p_{n}, \nabla T_{n}\right) .
\end{aligned}
$$

Here, $\Delta_{L}=\nabla^{T} \cdot(\nabla)$ and $\alpha_{p r}$ is a projection scheme weighting factor. The pressure field becomes

$$
p_{n+1}=p_{n}+\delta p_{n+1}
$$

and the velocity field is corrected on that basis,

$$
\boldsymbol{u}_{* *}=\boldsymbol{u}_{*}-\frac{f(\Delta t)}{\alpha_{p r} \rho_{n}} \nabla \delta p_{n+1} .
$$

The density is updated with the thermal equation for an ideal gas mixture,

$$
\rho_{n+1}=\frac{p_{r e f}+p_{n+1}}{(R T)_{n}}
$$

and the final velocity vector becomes

$$
\boldsymbol{u}_{n+1}=\frac{\rho_{n}}{\rho_{n+1}} \boldsymbol{u}_{* *} \cdot
$$

On the basis of resulting flow field quantities at the new time-step $n+1$, the divergence constraint is updated. Then, enthalpy and species mass fractions can be computed, depending on quantities at time-step $n+1$. The CPM solution strategy realizes a maximum spatial and temporal order of accuracy $\mathcal{O}(2)$.

\section{The Acoustic Model for Tur- bulent Combustion Noise}

One of the main objectives of this work is to apply a combustion noise monopole source term based model, which was formulated by Mühlbauer et al. [33], to a combustor application case in order to predict absolute combustion noise levels. The basic underlying theory as well as the principle functioning of the sound source reconstruction algorithm, FRPM, which was combined with the combustion noise formulation by Grimm et al. [20], is described in the following section.

\subsection{The Source Term Formulation}

The basis for the source term formulation derivation is a pressure-density relation

$$
\frac{1}{c^{2}} \frac{D p}{D t}=\frac{D \rho}{D t}+\rho \Phi
$$

with a right hand side expression $\Phi$ from Candel et al. [6], reading

$$
\begin{aligned}
\Phi= & \frac{\dot{Q}}{\rho c_{p} T}+M \frac{D}{D t}\left(\frac{1}{M}\right)+\frac{1}{\rho c_{p} T}\left[\nabla \cdot \lambda \nabla T+\boldsymbol{\tau}_{\boldsymbol{\tau}}: \nabla \mathbf{u}\right. \\
& \left.-\sum_{\alpha=1}^{N_{s}} \rho Y_{\alpha} c_{p, \alpha} \mathbf{V}_{\alpha}^{D} \cdot \nabla T\right],
\end{aligned}
$$

with $\lambda$, the heat conductivity and $\mathbf{V}_{\alpha}^{D}$, the diffusion velocity of species $\alpha$. $\dot{Q}$ is the volumetric heat release rate and $M$ denotes the molecular weight. Equation (25) is recast by making use of an energy equation formulation, leading to a complete expression with the heat release represented by a function of temperature change,

$$
\Phi=\frac{1}{T} \frac{D T}{D t}-\frac{1}{\rho c_{p} T} \frac{D p}{D t}+M \frac{D}{D t}\left(\frac{1}{M}\right) .
$$

According to Mühlbauer et al. [33], this formulation is a complete representation of the source term as given by Eqn. (25). Since usually applications with low Mach number flows are treated, the first term in Eqn. (26) is assumed to be dominant over the second, representing the effects of turbulent velocity fluctuations and the second term is therefore neglected. The third term in Eqn. (26) comes into picture only if the average molecular weight of combustion products significantly differs from the educts and is therefore also not considered here for the application to methane-air combustion systems.

Based on the first term of Eqn. (26), a right hand side processing rule for the source term formulation was obtained by transferring a pressure equation formulation

$$
\frac{D p}{D t}+\gamma p \nabla \cdot \mathbf{u}=\gamma p \Phi
$$

with the isentropic exponent $\gamma=c_{p} / c_{v}$, to the pressure equation form of the linearized Euler equations [33]. This procedure gives $q_{p}=(\gamma p \Phi)^{\prime}=\rho c^{2} \Phi-\overline{\rho c^{2} \Phi}$, which is then applied to Eqn. (26). The resulting, temperature variance based source term expression, which is subject to stochastic sound source reconstruction, reads

$$
q_{p}=\frac{\gamma \bar{p}}{\tilde{T}} \frac{\tilde{D} T^{\prime \prime}}{D t} .
$$

For the application of the full scale laboratory combustor simulation, it is coupled with a modified set of linearized Euler equations

$$
\begin{aligned}
& \frac{\partial \rho^{\prime}}{\partial t}+\widetilde{\boldsymbol{u}} \cdot \nabla \rho^{\prime}+\bar{\rho} \nabla \cdot \boldsymbol{u}^{\prime}=0, \\
& \frac{\partial \boldsymbol{u}^{\prime}}{\partial t}+(\widetilde{\boldsymbol{u}} \cdot \nabla) \boldsymbol{u}^{\prime}+\frac{\nabla p^{\prime}}{\bar{\rho}}=\mathbf{0}, \\
& \frac{\partial p^{\prime}}{\partial t}+\widetilde{\boldsymbol{u}} \cdot \nabla p^{\prime}+\gamma \bar{p} \nabla \cdot \boldsymbol{u}^{\prime}=q_{p},
\end{aligned}
$$


The source term in Eqn. (28) is subject to stochastic sound source reconstruction. Therefore, the Fast Random Particle Method for Combustion Noise Prediction (FRPM-CN), as introduced by Grimm et al. $[20]$ is employed. Sound sources are built from convected white noise at each time-step according to local turbulence statistics from CFD simulations. The spatial filtering algorithm of sources can be generally described by

$$
Q(\boldsymbol{x}, t)=\int_{V_{s}} \hat{A}(\boldsymbol{x}) \mathcal{G}\left(\left|\boldsymbol{x}-\boldsymbol{x}^{\prime}\right|, l_{T}(\boldsymbol{x})\right) \mathcal{U}\left(\boldsymbol{x}^{\prime}, t\right) d^{3} \boldsymbol{x}^{\prime} .
$$

A Gaussian shaped filter $\mathcal{G}$ is convoluted with a white noise field $\mathcal{U}$. The FRPM grid is orthogonal and therefore highly efficient Purser filters [37, 38] are employed for the source filtering, represented by $\mathcal{G}$. Integration of source components is performed over the source volume $V_{s}$ and the local amplitude scaling is realized according to $\hat{A}=\sqrt{\hat{R}(\boldsymbol{x}) / l_{T}^{3}(\boldsymbol{x})}$, in order to achieve the appropriate sound source variance.

The white noise field $\mathcal{U}$ is realized in a discrete form by mapping random values carried by floating particles onto a source field grid. This is done in FRPM, where particles are homogeneously seeded into the source region. Therefore, different kinds of flow field characteristics can be considered in the source region, like recirculation zones. This is a key aspect of the method and a decisive advancement compared to a preceding approach, RPM-CN [33], where the source field is discretized based on CFD RANS flow field streamlines. Those advancements in source discretization and the use of highly efficient filtering algorithms make 3D FRPM-CN an accurate, universally applicable and computationally efficient tool for combustion noise source modeling in complex test cases.

Besides the incorporation of local sound source extensions due to integral length-scales, turbulence effects have to be considered. Therefore, the spatial white noise field $\mathcal{U}$ is processed with a first order Langevin approach [14] in time for the realization of turbulence induced decay,

$$
\frac{D_{0}}{D t} \mathcal{U}=-\frac{1}{\tau_{T}} \mathcal{U}+\sqrt{\frac{2}{\tau_{T}}} \xi(\boldsymbol{x}, t) .
$$

Equation (33) is a stochastic differential equation, realizing a long-term drift behavior with the first component on the right hand side, while the second - so called diffusion term - introduces a Gaussian distributed white noise forcing, for which the random values have to be chosen appropriately [12] $D_{0} / D t=\partial / \partial t+\boldsymbol{u}_{0}^{c} \cdot \nabla$ and $\boldsymbol{u}_{0}^{c}$ is the mean CFD RANS velocity field. For $\xi(\boldsymbol{x}, t)$, the properties

$$
\begin{aligned}
\langle\xi(\boldsymbol{x}, t)\rangle & =0, \\
\langle\xi(\boldsymbol{x}, t) \xi(\boldsymbol{x}+\boldsymbol{r}, t+\tau)\rangle & =\delta\left(\boldsymbol{r}-\boldsymbol{u}_{0}^{c} \tau\right) \delta(\tau)
\end{aligned}
$$

hold. The brackets \langle\rangle denote ensemble-averaging. For small separation distance $\boldsymbol{r}$ and time $\tau$, the noise field $\mathcal{U}$ correlation can be expressed by taking into account the solution of Eqn. (33) and the source statistics from Eqn. (35),

$$
\langle\mathcal{U}(\boldsymbol{x}, t) \mathcal{U}(\boldsymbol{x}+\boldsymbol{r}, t+\tau)\rangle=\delta\left(\boldsymbol{r}-\boldsymbol{u}_{0}^{c} \tau\right) \exp \left(-|\tau| /_{T}\right),
$$

or in words, the Langevin process induces an exponential decorrelation. The overall FRPM-inherent correlation function, resulting from the Langevin-induced decorrelation and the use of a Gaussian-shaped filter for the sound reconstruction procedure resulting from Eqn. (32), reads

$$
\mathcal{R}(\boldsymbol{x}, \boldsymbol{r}, \tau)=\hat{R}(\boldsymbol{x}) \exp \left(-\frac{|\tau|}{\tau_{T}}-\frac{\pi}{4 l_{T}^{2}(\boldsymbol{x})}\left|\boldsymbol{r}-\boldsymbol{u}_{0}^{c} \tau\right|^{2}\right) .
$$

Equation (37) is used as the correlation function of combustion noise sources for the presented numerical simulations with $\hat{R}(\boldsymbol{x})=\widetilde{T^{\prime \prime 2}}(\boldsymbol{x}) / \tau_{T}(\boldsymbol{x})^{2} . \quad \boldsymbol{r}$ and $\tau$ in Eqn. (37) are the separation distance and time, respectively, while $\tau_{T}$ and $l_{T}$ are the local turbulent time- and length-scale.

The source term formulation of Eqn. (28) is explicitly realized in FRPM-CN by

$$
\begin{aligned}
& \mathcal{R}(\boldsymbol{x}, \boldsymbol{r}, \tau)=\left\langle q_{p} q_{p}\right\rangle= \\
& \quad=\left(\frac{\gamma \bar{p}}{\tilde{T}}\right)^{2}\left\langle\frac{\tilde{D} T^{\prime \prime}}{D t}(\boldsymbol{x}, t) \frac{\tilde{D} T^{\prime \prime}}{D t}(\boldsymbol{x}+\boldsymbol{r}, t+\tau)\right\rangle \\
& \quad=\left(\frac{\gamma \bar{p} \widetilde{T}^{\prime \prime}}{\tilde{T} \tau_{T}}\right)^{2} \exp \left(-\frac{|\tau|}{\tau_{T}}-\frac{\pi}{4 l_{T}^{2}(\boldsymbol{x})}\left|\boldsymbol{r}-\boldsymbol{u}_{0}^{c} \tau\right|^{2}\right) .
\end{aligned}
$$

The convective part of the substantial derivative in Eqn. (28) is incorporated in Eqn. (38) by the argument of the exponential function $\left|\boldsymbol{r}-\boldsymbol{u}_{0}^{c} \tau\right|^{2}$, which comes from the properties of convected noise, as introduced with Eqns. (35) and (36).

Whether the employed two-point space-time correlation function is suitable for the modeling of combustion noise source dynamics, is evaluated in the CCA Results section by comparison of pressure spectra with experimental data. 


\section{Combustor Application Case}

The main objective of this work is broadband combustion noise prediction in a laboratory-scale combustor with the hybrid 3D FRPM-CN method and the comparison with a scale-resolving, compressible approach. In the following section, the basic configurations of CFD and CCA (Computational Combustion Acoustics) simulations are introduced. The burner is well characterized from an experimental point of view. Velocity profiles for the validation of CFD simulations are available from PIV measurements conducted by Stöhr et al. [42]. The temperature profiles were recorded with Raman spectroscopy by Meier et al. [30] and Weigand et al. [48]. Acoustic pressure was recorded in the combustion chamber at the positions shown in Fig. 4. Calibrated microphone probes with B\&K Type 4939 condenser microphones were used recording with a sampling rate of $100 \mathrm{kHz}$ [42]. A sketch of the combustor is depicted in Fig. 2. The main difficulties for the simulation of flow and combustion of this model combustor are flame lift off as well as flow detachment in the region of the curved surface forming the exit of the burner. Both phenomena are crucial for the successful simulation of this particular application case. The inves-

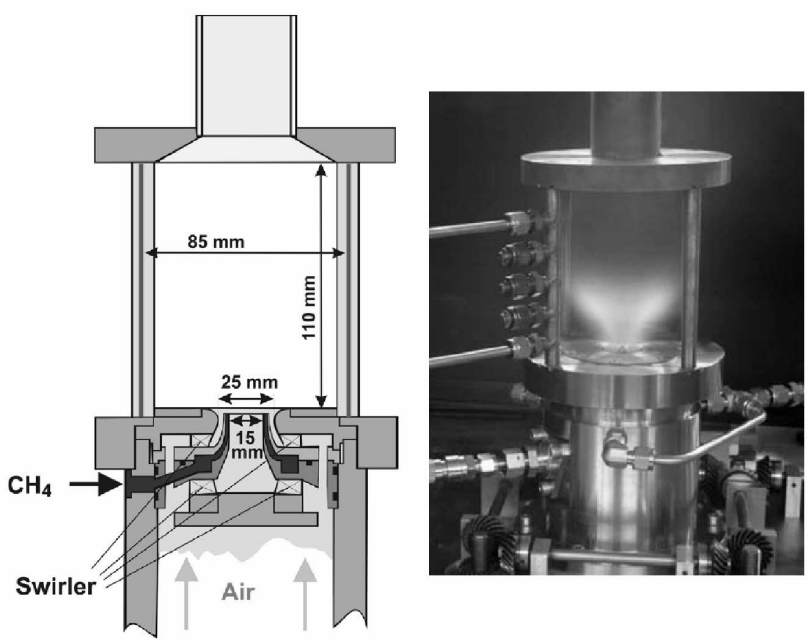

Figure 2: Schematic Drawing and Operation of the Combustor with Basic Dimensions [48].

tigated reference case is operated at a thermal power of $P_{\mathrm{th}}=34.9 \mathrm{~kW}$ with equivalence ratio and mixture fraction of the overall mixture of $\Phi=0.65$ and $f=0.037$. A stable operation case is treated with no thermo-acoustic behavior observed. The Reynolds number amounts to 52500, determined at the airplenum inlet and the swirl number of the combustor is 0.9 [48]. Air is induced into an upstream air-plenum with $\dot{m}_{\text {air }}=0.01825 \mathrm{~kg} / \mathrm{s}$ and is then internally separated to approach the combustion chamber through an inner and an outer radially aligned swirler, while the fuel is induced between the two swirled air flow streams with $\dot{m}_{\text {fuel }}=0.0007 \mathrm{~kg} / \mathrm{s}$. Due to this align- ${ }_{333}$ ment, the burner operates in a partially premixed mode at atmospheric conditions.

\subsection{CFD Setup}

The computational setup of the reacting CFD simulations was introduced extensively by Reichling et al. [40] and is therefore only sketched here. The employed grid is shown in Fig. 3. CFD RANS simulations were also conducted on the grid of the SAS setup. It consists of mainly tetrahedral elements with locally resolved near-wall regions, while prism layers and tetrahedral elements are connected via pyramids. A total of 11.3 million cells with 2.38 million grid nodes is used and the condition $y^{+} \approx 1$ is satisfied in refined regions of the swirler and combustion chamber walls. Simulations are carried out with the

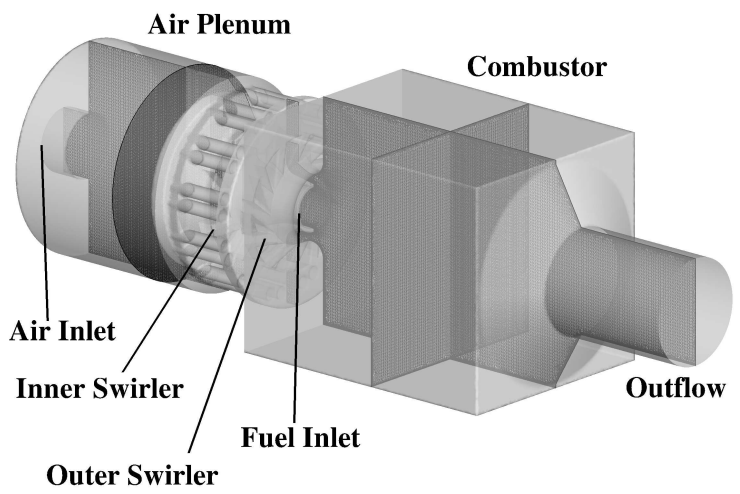

Figure 3: Unstructured CFD Mesh.

DLR finite volume based CFD code THETA [10]. For the incompressible steady state reacting RANS simulations, a SIMPLE (Semi-Implicit Method for Pressure Linked Equations) solution strategy is employed. The $k-\omega$-SST turbulence model in a formulation of Menter [32] is used and reactions are modeled with a global ansatz, taking into account a three-step formulation of methane combustion with air proposed by Nicol et al. [35]. An additional transport equation for the temperature variance is solved (Eqn. (16)) and the resulting field is used for the reconstruction of the temperature variance based combustion noise source term. Direct simulations are conducted with the $k-\omega$-SST SAS model and Projection Method based solver schemes. Also for the SAS simulations, global chemistry treatment is used, as introduced previously. The SAS approach was derived by Menter and Egorov $[31,11]$ and is essentially a hybrid URANS/LES formulation.

In total three different simulations are carried out. Specifications are listed in Table 1. 
Table 1: Specifications of CFD simulations. CPM: Compressible Projection Method [40], IPM: Incompressible Projection Method.

\begin{tabular}{cccc}
\hline Mode & Solver & Turbulence & $\Delta t$ \\
\hline RANS & SIMPLE & $k$ - $\omega$-SST & - \\
SAS & IPM & $k-\omega$-SST SAS & $2.5 \cdot 10^{-7}$ S \\
SAS & CPM & $k-\omega-$ SST SAS & $1.0 \cdot 10^{-6}$ S
\end{tabular}

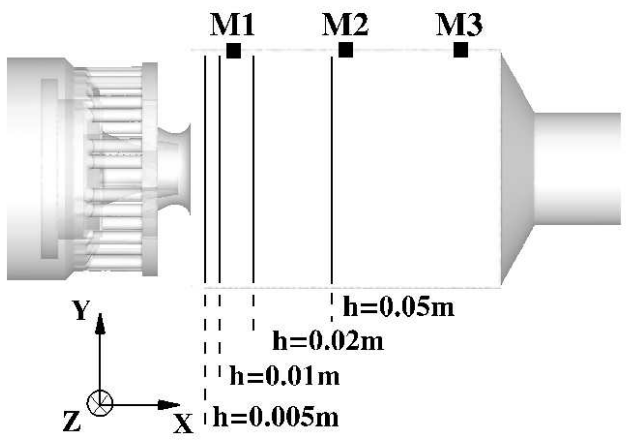

Figure 4: Positions of Profile Lines for Experimental Flow Field and Combustion Data and Acoustic Pressure Recorder Positions in the Combustor (M1-M3).

The acoustics simulations are carried out with the DLR inhouse CAA (Computational Aero-Acoustics) code PIANO, including the FRPM module for stochastic sound source reconstruction. Sound propagation in space is computed via a dispersion relation preserving (DRP) scheme from Tam \& Webb [46]. For progression in time, a low-dissipation, low-dispersion four step Runge-Kutta scheme [22] is employed. The computational combustion acoustics grid is shown in

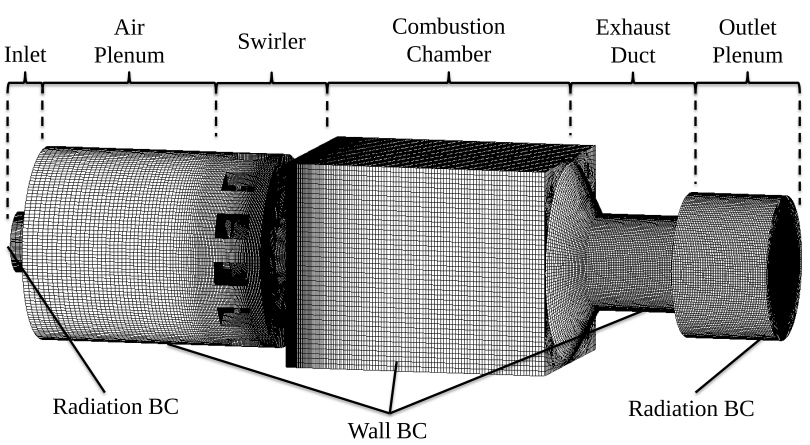

Figure 5: Computational Combustion Acoustics Grid With Employed Boundary Conditions.

Fig. 5. The grid for the acoustics simulations is optimized with respect to the local growth rate of adjacent cells, since the finite difference DRP scheme requires smooth grid transitions. The air plenum is simplified, since the tube-connectors between the two swirlers would lead to very fine cells, due to a time step limit of $t_{\mathrm{lim}}=\left(2.83 l_{\mathrm{min}}\right) /(\pi(1+\mathrm{Ma}))$ holding for stability reasons. The mesh is block-structured. It consists of $5.85 \cdot 10^{6} 3 \mathrm{D}$ hexahedral elements with $7.52 \cdot 10^{6}$ nodes, distributed to 2696 blocks. The highest spatial resolution of the mesh is given in the regions around the tip of the averaged flame front, close to the swirler nozzle exit. A minimum lengthscale of $l_{\min }=9 \cdot 10^{-4} \mathrm{~m}$ with four discrete points per length is resolved in each spatial direction. The maximum growth rate of adjacent cells in critical regions is $5 \%$ and the spatial resolutions then result from the combustor blocking in combination with the use of a seven-point finite difference stencil.

Mean flow field solutions for $\bar{\rho}, \tilde{u}, \tilde{v}, \tilde{w}, \bar{p}$ from the preceding CFD RANS simulations are interpolated onto the CCA grid via a statistical Kriging [27] algorithm. Inlet boundaries are treated with a radiation condition from Tam \& Webb [46] and the combustor walls are modeled with the ghost point concept of Tam \& Dong [45]. In the experiments, the combustor flow expands to an exhaust duct after a rapid acceleration and a following outlet tube. In the numerical simulation, an additional plenum is attached to the tube, in order to enforce a natural tube impedance exit with base flow. The outlet plenum in turn is surrounded by non-reflecting radiation boundary conditions and a damping sponge-layer is superimposed.

Pressure sensors in the combustion chamber are located in the corner rails in the experiments, which are holding the optical access glass walls. Their positions are indicated in Fig. 4. All dimensionless quantities are referenced to air plenum atmospheric conditions. Source region extensions in the numerical simulation are chosen by means of a discrete realization of Eqn. (28), reading

$$
\varphi=\frac{\gamma \bar{p}}{T} \cdot \sqrt{\frac{T^{\prime \prime 2}}{\tau_{T}^{2}}} .
$$


As can be seen from Eqn. (38), the indicator $\varphi$ in Eqn. (39) represents a discrete form of the combustion noise source term for vanishing spatial and temporal separation. Or, in other words, it indicates regions in the combustion chamber where combustion noise sources according to Eqn. (28) are present. It is evaluated from the CFD RANS field solution and its profile on a combustor mid-plane is shown in Fig. 6 .

Source field extensions are chosen accordingly. $\varphi$ is normalized to its maximum value in the inner shear layer and values smaller than $5 \%$ are not considered. Two source regions are employed, while the reconstructed sources on each source field are weighted with sine-functions in the overlapping areas,

$$
W(x)=\underbrace{\sin ^{2}(x)_{\mathrm{S} 1}}_{W_{\mathrm{S} 1}(x)^{2}}+\underbrace{\cos ^{2}(x)_{\mathrm{S} 2}}_{W_{\mathrm{S} 2}(x)^{2}}=1 .
$$

The weighting functions with overlapping sound sources are displayed in Fig. 7. Weighting is applied to the temperature variance field solution in the source region, which accounts for local amplitude scaling of combustion noise source fluctuation. However, sound source magnitudes are evaluated in FRPM-CN by using the standard deviation of temperature. Therefore, sine and cosine functions are applied to the variance field, so that a consistent value of unity according to Eqn. (40) is conserved in the whole source region. This method is employed for efficiency reasons. Specifications of S1 are $x \in[-0.004 \mathrm{~m} ; 0.008 \mathrm{~m}], y, z \in[-0.024 \mathrm{~m} ; 0.024 \mathrm{~m}]$ and for S2 $x \in[0.002 \mathrm{~m} ; 0.045 \mathrm{~m}], y, z \in[-0.038 \mathrm{~m} ; 0.038 \mathrm{~m}]$. Both source fields are discretized with a minimum

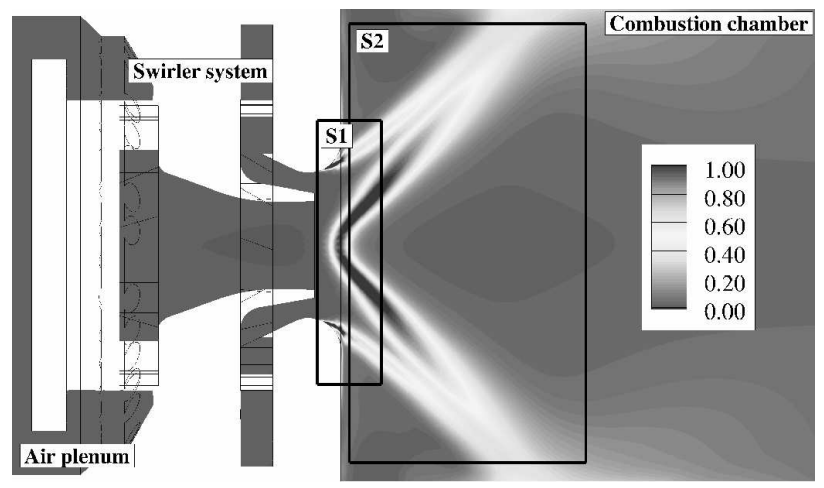

Figure 6: Distribution of contributions to combustion noise according to Eq. (39), evaluated from CFD RANS quantities and normalized to the inner shear layer maximum value.

resolution of $l_{\min }=3 \cdot 10^{-3} \mathrm{~m}$, with four discrete points per length and $\Delta x=\Delta y=\Delta z$. A numerical time step of $\Delta t=1.4 \cdot 10^{-7} \mathrm{~s}$ is used for the overall CCA numerical simulations, which is limited by the smallest CCA-grid cell rather than the sound source reconstruction algorithm in this particular case.
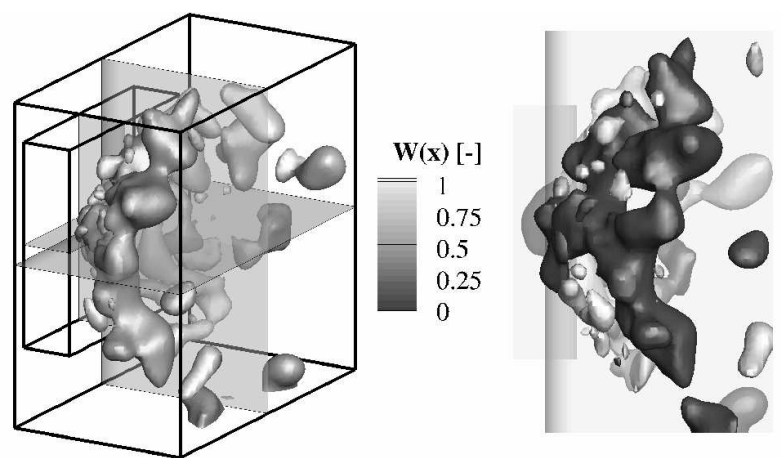

Figure 7: Depiction of employed weighting functions $W_{S 1}(x), W_{S 2}(x)$ on the source fields S1 and S2 with exemplary isocontours of resulting combustion noise sound sources. $W(x) \in[0 ; 1]$.

\section{$5 \quad$ Numerical Results}

In the following section, selected results from the CFD simulations with THETA and the combustion acoustics simulations with PIANO and THETA (CPM) are shown.

\subsection{CFD Results}

A steady-state reacting CFD RANS and two unsteady $k$ - $\omega$-SST-SAS simulations (IPM and CPM) of flow field and combustion are analyzed. The computational costs for a CFD RANS calculation are $2.1 \cdot 10^{3}$ CPU-hours, run on $4 * 8$ Nehalem cores, while the incompressible $k$ - $\omega$-SST-SAS simulation is conducted in $3.2 \cdot 10^{4}$ CPU-hours on $4 * 8$ Nehalem cores, where the total simulation time is ten combustor residence times. The compressible $k$ - $\omega$-SST-SAS simulation amounts to $1.4 \cdot 10^{5}$ CPU-hours on $16 * 16$ SandyBridge cores for the same number of residence times, due to a smaller time-step compared to the IPM simulation, which is needed for stability reasons.

Averaged $x$ and $y$ components of velocity, average temperature and RMS of temperature along several profile lines, as shown in Fig. 4, are superimposed with the respective experimental results in Figs. 8, 9, 13 and 14. Furthermore, the degree of resolution of turbulence in $k$ - $\omega$-SST-SAS simulations is analyzed in Figs. 10 and 11 . This is done by evaluating the ratio of turbulent to laminar viscosity $r_{\mu}$ and the ratio of resolved to overall kinetic energy $r_{k e}$. Those two criteria not only assess the turbulence resolution in a LES-like simulation but also the region where the SAS model operates in LES-like mode and where URANS modeling takes place.

Regarding the viscosity ratio, values lower than 20 are achieved in the combustion chamber, while larger values are present in the shear layers of the swirling flow. Values are slightly higher for the CPM simulation, especially close to the flame root in the inner shear layer. Large viscosity ratios and small kinetic energy ratios 
in the upstream air plenum indicate that the simulation works in URANS mode in those regions. At least $80 \%$ of turbulent motion are directly resolved within the combustion chamber for both, IPM and CPM, as indicated by $\mu_{k e}$ in Figs. 10 and 11. Therefore, it can be stated that turbulence is fairly well resolved by the $k$ - $\omega$-SST-SAS simulation in the combustion chamber, where reaction takes place and combustion noise sources are located. Furthermore, an exemplary spectrum of resolved turbulent kinetic energy in the combustion chamber of the CPM simulation is shown in Fig. 12. Turbulent fluctuation is well resolved over a large range of frequencies and the shape of the spectrum for higher frequencies follows the $-5 / 3$-slope, which is however based on isotropic turbulence. A peak in the energy spectrum at $1690 \mathrm{~Hz}$ indicates a helical vortex instability [42]. This flow feature emerges in the swirler and propagates a periodic cascade of vortices into the combustion chamber. It is reflected in the sound pressure spectra of experiment and CPM in Fig. 16.

Axial $x$ - and $y$-velocity profiles in Fig. 8 and Fig. 9, according to positions in Fig. 4, show typical flow field characteristics of a swirl-stabilized flow. The flow expands in a v-shaped manner, while a lowpressure region, the so-called inner recirculation zone, forms along the center-line burner axis, where negative mean $x$-velocities are present. $y$-velocities are zero along the center-line axis, due to rotational symmetry of the swirled flow. Furthermore, due to the sharp expansion of the flow geometry, outer recirculation zones develop.

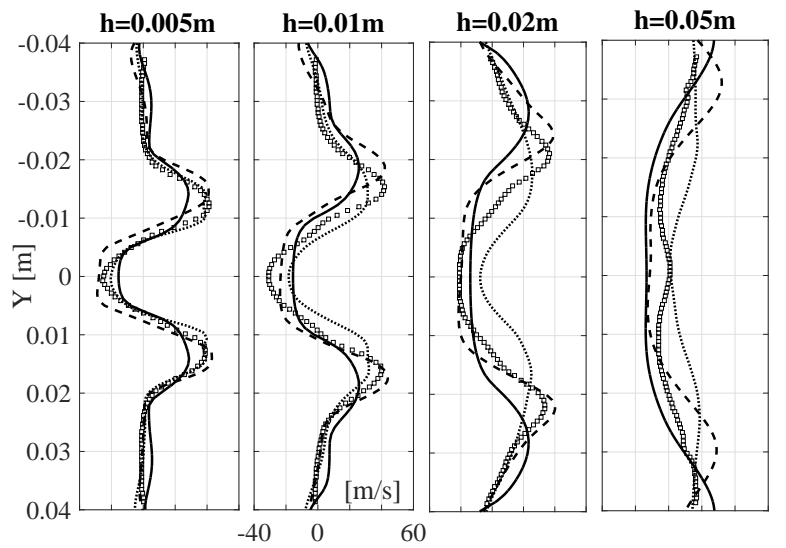

Figure 8: Experimental profiles [42] of axial $x$-velocity $\left[\frac{\mathrm{m}}{\mathrm{s}}\right](\square)$, CFD RANS (continuous), IPM (dashed) and CPM (dotted) simulation results.

The steady state CFD-RANS simulations reveal relatively good agreement with the experimental profiles for all downstream line positions, while the computational effort is one order of magnitude lower than that of the SAS calculations. However, two distinct deviations to the experimental profiles can be observed in Figs. 8 and 9: A too-wide opening angle of the flow

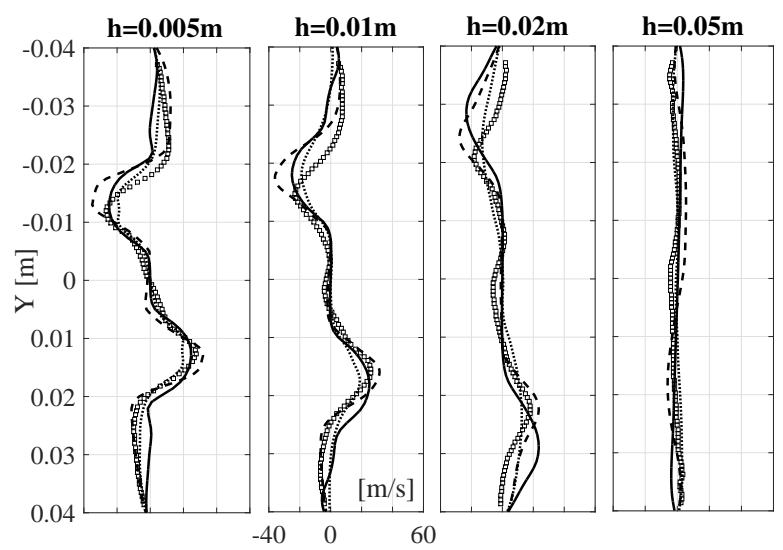

Figure 9: Experimental profiles [42] of $y$-velocity [ $\left.\frac{\mathrm{m}}{\mathrm{s}}\right]$ ( $\square)$, CFD RANS (continuous), IPM (dashed) and CPM (dotted) simulation results.

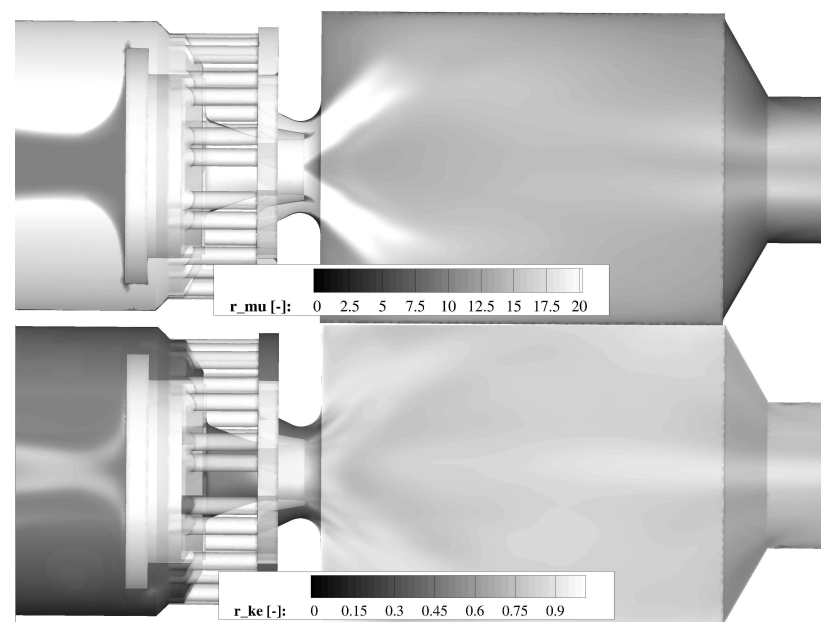

Figure 10: Turbulence resolution of the IPM $k$ - $\omega$-SST-SAS simulation, ratio of viscosities $r_{\mu}=\mu_{t} / \mu$ and ratio of resolved to overall kinetic energy $r_{\mathrm{ke}}=k_{\text {res }} / k_{\text {overall }}$. 


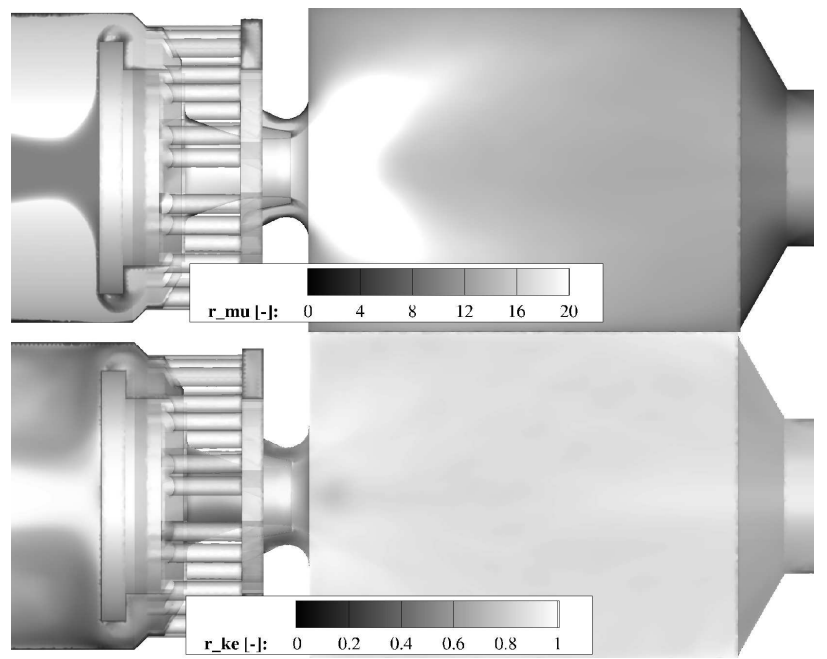

Figure 11: Turbulence resolution of the CPM $k$ - $\omega$-SST-SAS simulation, ratio of viscosities $r_{\mu}=\mu_{t} / \mu$ and ratio of resolved to overall kinetic energy $r_{\mathrm{ke}}=k_{\text {res }} / k_{\text {overall }}$.

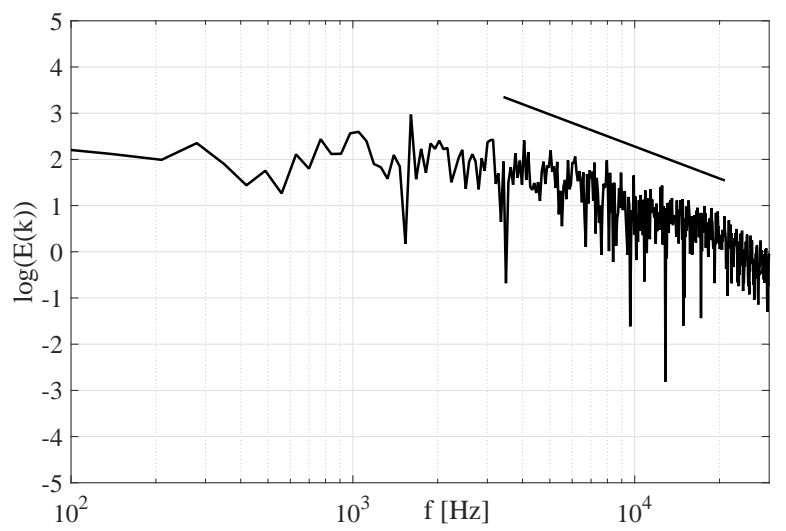

Figure 12: Spectrum of turbulent kinetic energy of the CPM $k$ - $\omega$-SST-SAS simulation, evaluated in the inner shear layer. Indicated idealized $-5 / 3$ slope.

Frequency over logarithmic kinetic energy in

$$
\log \left[m^{2} / s^{2}\right] \text {. }
$$

and an under-prediction of the maximum values of the axial velocity component. The under-prediction of peak values in axial velocity is a result of the large opening angle of the swirled flow. Nonetheless, due to our experience, the achieved quality of the RANS flow field is sufficient to provide an adequate base for the convective movement of later reconstructed sound sources.

The unsteady IPM and CPM $k$ - $\omega$-SST-SAS both capture the flow-field very well. However, the IPM simulation tends to over-predict the flow opening angle, similarly to the RANS calculation, but nicely reproduces peak values. The CPM simulation underpredicts flow opening angles for downstream positions and slightly under-represents absolute values for the profile peaks, especially for axial velocity.

In total, the SAS gives a clearly better representation in terms of absolute values and the mean flow field in the inner recirculation zone.

Temperature and temperature rms profiles are shown in Figs. 13 and 14, according to positions in Fig. 4. In accordance to the mean flow field, the temperature of RANS and IPM profiles reveal a too-large opening angle. As a second distinct characteristic, the lift-off height of the flame is too low and therefore temperature levels at profile lines close to the combustor inlet are over-predicted in the case of RANS and IPM. This might be caused by an over-prediction of reaction rates close to the inner shear layer, due to the use of an EDM/FRC model in combination with global chemistry treatment. A further reason for this could be the lack of accountancy for turbulence-chemistry interaction, since this deviation can be observed in both, RANS and URANS/LES simulation. CPM

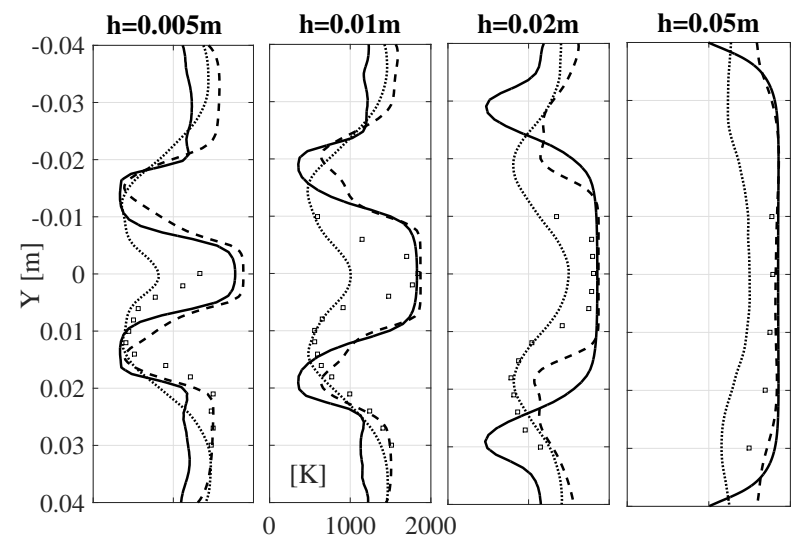

Figure 13: Experimental temperature profiles $[30,48][\mathrm{K}](\square)$, CFD RANS (continuous), IPM (dashed) and CPM (dotted) simulation results.

gives a better representation of profile shapes of temperature and temperature RMS. However, absolute values are significantly under-predicted. This is due to the flame anchoring further downstream in case of a CPM simulation. Maximum values of temperature 


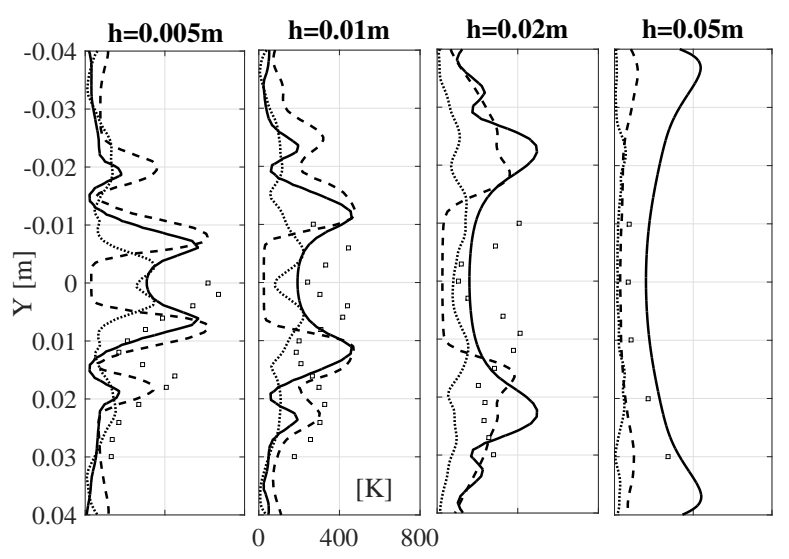

Figure 14: Experimental temperature RMS profiles $[30,48][\mathrm{K}](\square)$, CFD RANS (continuous), IPM (dashed) and CPM (dotted) simulation results.

RMS are nicely captured in the numerical simulation of RANS and IPM. Temperature RMS profiles of the RANS simulation are used for amplitude scaling of fluctuation of combustion acoustics sources in the hybrid approach. Since peak values are in good agreement with experiments, it is expected to reproduce correct sound pressure amplitudes. The steady state reacting RANS simulation therefore seems to perform satisfactory.

\subsection{CCA Results}

In preceding works, detailed validation of FRPM-CN was carried out for jetflames [20]. In that context, coupling constants were derived as model parameters of the stochastic sound source reconstruction algorithm. In the presented work, no specific adaption is undertaken.

Volumetric sound source reconstruction is performed at every time step based on turbulence statistics from preceding CFD RANS simulations. Exemplary results of these calculations are shown for instantaneous fluctuating acoustic pressure and sound sources in Fig. 15. No spurious reflection is observed with the employment of non-reflecting radiation conditions for the boundaries of the combustor outlet plenum, following the exhaust tube. For the choice of outlet plenum spatial extensions it was accounted for an approximated impedance induced end correction in axial direction based on investigations of Munt [34], da Silva et al. [8] and a formulation of Davies et al. [9], for which the local speed of sound was taken into consideration. FRPM-CN sound pressure spectra are compared to experimental data [43] and the compressible CPM SAS simulation in Fig. 16. Experiments indicate a weak thermoacoustic oscillation at about $350 \mathrm{~Hz}$ and a second peak at $1690 \mathrm{~Hz}$, caused by a helical instability [42]. FRPM-CN spectra in Fig. 16 are based on the source term expression in Eqn. (28). Absolute levels are captured well with the hybrid method, especially

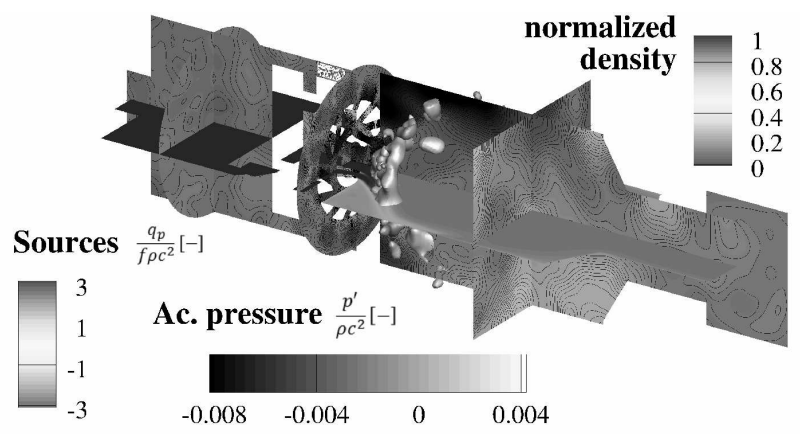

Figure 15: Combustor midplane cuts of instantaneous sound pressure, CFD RANS density distribution (horizontal cut plane) and exemplary isosurfaces of combustion acoustic sources.

for low frequencies. Sound pressure levels are computed without any artificial scaling. Therefore, 3D FRPM-CN quantitatively predicts combustion noise emission in swirl stabilized combustion systems, relying on CFD RANS input only. The approach delivers feasible results for combustion induced noise prediction, despite certain inaccuracies of the underlying CFD-RANS simulations. Over-prediction of levels in the region of $400 \mathrm{~Hz}$ to $2000 \mathrm{~Hz}$ can be linked to poor reproduction of temperature variance profiles from Eqn. (16), especially in the inner recirculation zone as shown in Fig. 14. This leads to the formation of unwanted source components, where no temperature fluctuation is present in the experiment.

The SAS simulation with the Compressible Projection Method (CPM) also reproduces sound pressure levels in the combustion chamber with consistency for all investigated microphone positions. Nonetheless, low frequency levels are slightly under-predicted and the hybrid method seems to be superior for lower frequencies. This might be linked to the compressible SAS not entirely resolving turbulent motion close to the inner recirculation zone, as indicated in Fig. 11.

CPM captures the helical flow instability at $1690 \mathrm{~Hz}$ in the presure spectrum shown in Fig. 16a. This phenomenon is also detectable in the CPM turbulence spectrum in Fig. 12. It cannot be depicted with the sequential, hybrid FRPM-CN. However, acoustical dynamics related to eigenmodes of the system are perceivable in FRPM-CN simulations, resulting in peaks in the numerically obtained spectra.

Absolute sound pressure levels predicted by the hybrid and the direct approach show similar levels for all the investigated measurement positions in the combustion chamber. This indicates that, in this particular case, direct combustion noise is dominant compared to indirect noise, since indirect noise dynamics are not depicted by the hybrid method.

Contributions to combustion noise in the combustion chamber are analyzed for the different simulations based on Eqn. (39) in Fig. 17. Profiles are evalu- 
ated from the modeled part of turbulence quantities and therefore show a qualitative statistical behavior, normalized to the respective maximum values in the inner shear layer. Sound sources are mainly located in the shear layers, as can be seen from the profiles in Fig. 17. There are significant contributions for both, inner and outer shear layers for all simulated cases. As reflected in the flow field and temperature profiles in Figs. 8, 9, 13, and 14, the opening angle of the flow is over-predicted in the RANS and IPM simulations and the flame stabilizes slightly too far upstream, compared to experimental data. Due to different locations of averaged combustion noise profile locations in Fig. 17, it can be stated that the exact average position of the flame is not crucial for reproducing experimental sound pressure spectra in Fig. 16, but rather the accurate prediction of absolute temperature variance peak levels is.

A total of $N_{C C A}=2.3 \cdot 10^{5}$ time steps and $\Delta t=$

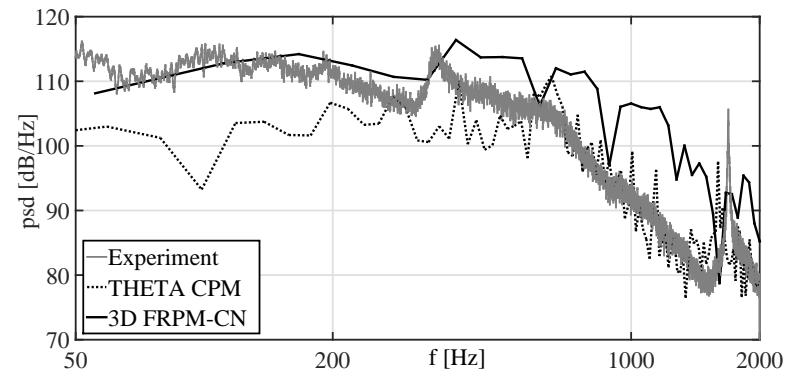

(a) Position $(x=0.015 \mathrm{~m}|y=0.042 \mathrm{~m}| z=0.042 \mathrm{~m})$.

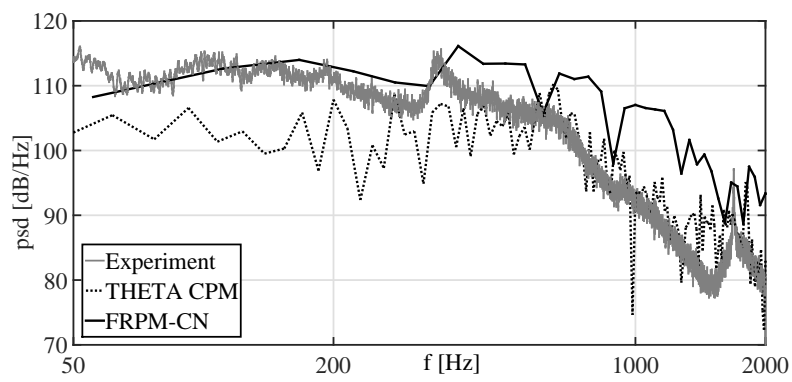

(b) Position $(x=0.055 \mathrm{~m}|y=0.042 \mathrm{~m}| z=0.042 \mathrm{~m})$.

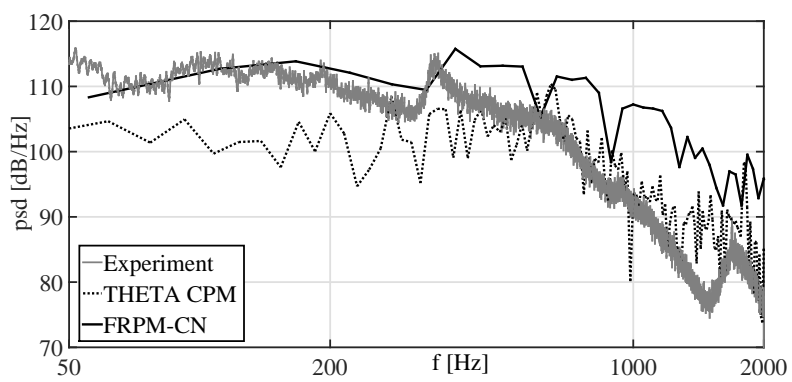

(c) Position $(x=0.095 \mathrm{~m}|y=0.042 \mathrm{~m}| z=0.042 \mathrm{~m})$.

Figure 16: Pressure spectra in the combustion chamber according to Fig. 2. Comparison between experiment, 3D FRPM-CN and a compressible projection method (CPM).
$1.4 \cdot 10^{-7} \mathrm{~s}$ is simulated with $3 \mathrm{D}$ FRPM-CN, computed on $3 * 24$ Nehalem cores in $1.52 \cdot 10^{4}$ CPU-hours. The fraction of CFD RANS computational time is $2.1 \cdot 10^{3}$ CPU-hours, making up $12.14 \%$ of the total computational effort.

On the contrary, the SAS simulations with IPM and CPM in THETA were conducted in $3.2 \cdot 10^{4}$ and $1.36 \cdot 10^{5} \mathrm{CPU}$-hours, respectively. Ten combustor residence times were simulated with $\Delta t=2.5 \cdot 10^{-7} \mathrm{~s}$ for $\mathrm{CPM}$, considering a significant amount of simulation time for convergence with a residence time amounting to $0.035 \mathrm{~s}$. This results in $N_{C P M}=1.4 \cdot 10^{6}$ computed time steps. Furthermore, due to the use of different node architectures, the hybrid approach 3D FRPM$\mathrm{CN}$ is more than one order of magnitude faster than the investigated direct approach.

However, 3D FRPM-CN lacks the possibility to account for thermoacoustic phenomena or the depiction of the flow-instability as seen herein, which periodically influences the flame and results in a spectral peak.

\section{Conclusions}

In the presented paper, a detailed comparison of a hybrid and a direct approach for the simulation of combustion acoustics in a laboratory scale combustor featuring partially premixed, swirl stabilized combustion was carried out. The hybrid method is based on turbulent statistic quantities which were taken from a steady RANS method. Experimental data for the mean flow field and combustion as well as two SAS simulations with different solvers were used for the validation and performance evaluation of the hybrid approach. Sound pressure spectra were compared to experimental data and simulation results of a compressible projection scheme (CPM) simulation. The results of the presented work revealed that CFDRANS simulations provided a reasonable flow field and temperature distribution by little computational effort but were inferior to LES/URANS results, as expected.

The solution of an additional transport equation for the temperature variance distribution on top of CFDRANS results with global chemistry modeling showed good agreement with experimental data in terms of peak values but showed discrepancies for the shape of overall profiles. It was demonstrated that both, 3D FRPM-CN with RANS and CPM with SST-SAS were capable of reproducing absolute sound pressure levels in the combustion chamber.

The hybrid method 3D FRPM-CN predicted the turbulent combustion noise spectrum in good agreement with experimental data in cases without strong thermoacoustics with efficient and robust models and is therefore highly suitable as a tool for the design of noise reduction measures in all kinds of technically 


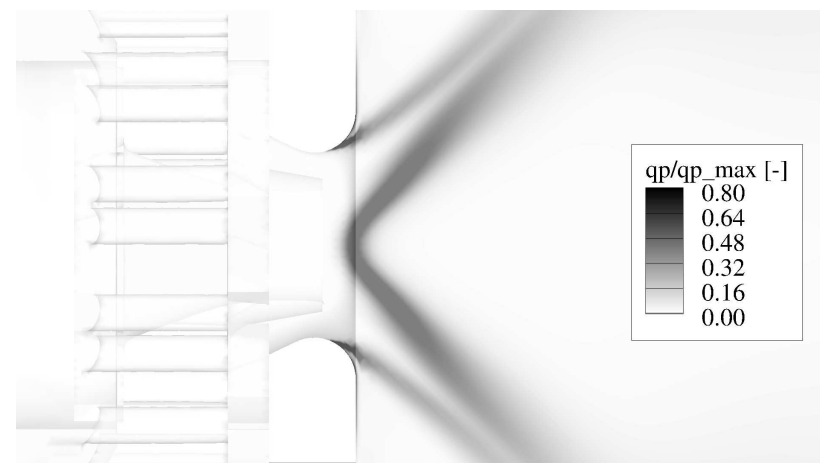

(a) RANS, $k-\omega$-SST.

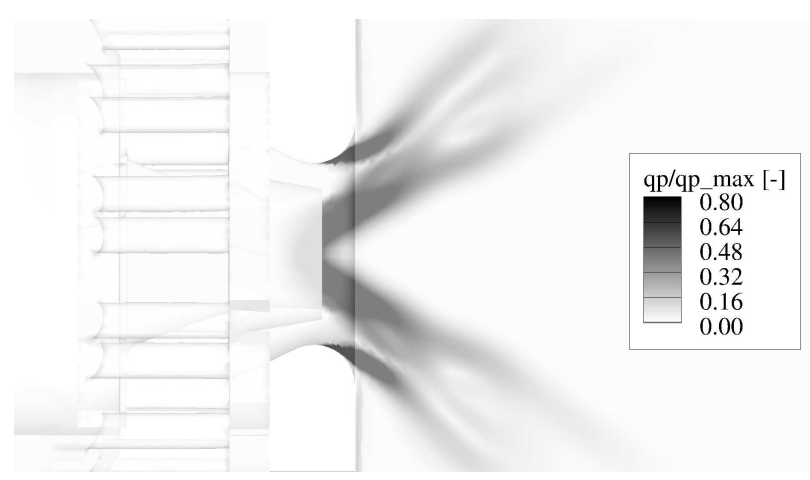

(b) IPM, $k$ - $\omega$-SST SAS.

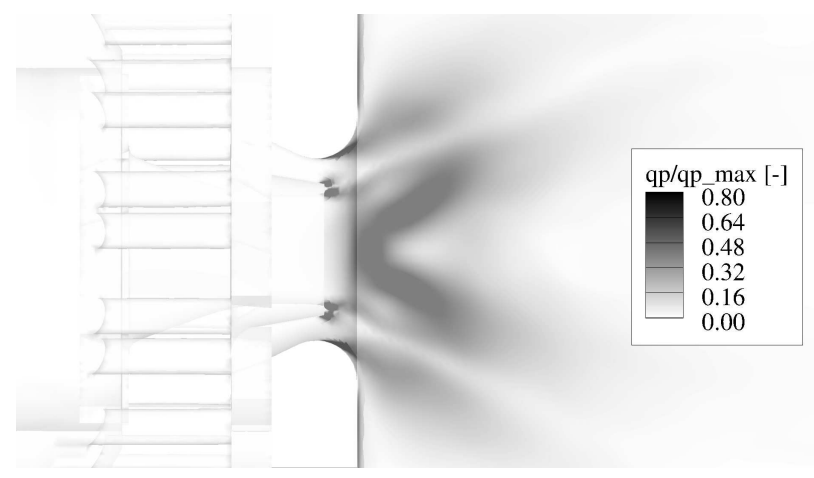

(c) CPM, $k$ - $\omega$-SST SAS.

Figure 17: Estimation of sound source location according to Eqn. (39) in the combustion chamber, based on CFD RANS, IPM and CPM $k-\omega$-SST-SAS simulation data.

can be monitored from a much earlier point.

\section{Acknowledgement}

This collaborative work was partially supported within the project DECISIVE which was funded by the German Aerospace Center (DLR).

\section{References}

[1] C. Bailly and D. Juvé. A Stochastic Approach To Compute Subsonic Noise Using Linearized Euler's Equations. In 5th AIAA/CEAS Aeroacoustics Conference, 1999. AIAA Paper 1999-1872.

[2] W. Béchara, C. Bailly, and P. Lafon. Stochastic Approach to Noise Modeling for Free Turbulent Flows. AIAA Journal, 32(3):455-463, 1994.

[3] M. Billson, L.-E. Eriksson, and L. Davidson. Jet Noise Prediction Using Stochastic Turbulence Modeling. In 9th AIAA/CEAS Aeroacoustics Conference, 2003. AIAA Paper 2003-3282.

[4] H. Brick, R. Piscoya, M. Ochmann, and P. Költzsch. Prediction of the sound radiation from open flames by coupling a large eddy simulation and a kirchhoff method. Acta Acustica united with Acustica, 91:17-21, 2005.

[5] T.Ph. Bui, W. Schröder, and M. Meinke. Numerical analysis of the acoustic field of reacting flows via acoustic perturbation equations. Computers Es Fluids, 37(9):1157-1169, 2008.

[6] S.M. Candel, D. Durox, S. Ducruix, A.-L. Birbaud, N. Noiray, and T. Schuller. Flame dynamics and combustion noise: progress and challenges. International Journal of Aeroacoustics, 8(1):1-56, 2009.

[7] A. J. Chorin. A numerical method for solving incompressible viscous flow problems. Journal of Computational Physics, 2:12-26, 1967.

[8] A.R. da Silva, G.P. Scavone, and A. Lefebvre. Sound reflection at the open end of axisymmetric ducts issuing a subsonic meanflow: A numerical study. Journal of Sound and Vibration, 327:507$528,2009$.

[9] P.O.A.L. Davies, J.L. Bento Coelho, and M. Bhattacharya. Reflection coefficients for an unflanged pipe with flow. Journal of Sound and Vibration, 72(4):543-546, 1980.

[10] M. Di Domenico, P. Gerlinger, and B. Noll. Numerical simulations of confined, turbulent, lean, premixed flames using a detailed chemistry combustion model. In Proceedings of the ASME Turbo Expo 2011, 2011. GT2011-45520. 
[11] Y. Egorov and F. Menter. Development and application of sst-sas turbulence model in the desider project. In In: Advances in Hybrid RANS-LES Modelling, 2008. Springer, pp. 261270.

[12] R. Ewert. RPM - the fast random particlemesh method to realize unsteady turbulent sound sources and velocity fields for CAA applications. In 13th AIAA/CEAS Aeroacoustics Conference, AIAA 2007-3506, 2007.

[13] R. Ewert. Broadband Slat Noise Prediction Based on CAA and Stochasic Sound Sources from a Fast Random Particle-Mesh (RPM) Method. Computers and Fluids Journal, 37(4):369-387, 2008.

[14] R. Ewert, J. Dierke, J. Siebert, A. Neifeld, C. Appel, M. Siefert, and O. Kornow. CAA broadband noise prediction for aeroacoustic design. Journal of Sound and Vibration, 330(17):4139-4160, 2011.

[15] R. Ewert and R. Emunds. CAA Slat Noise Studies Applying Stochastic Sound Sources Based On Solenoidal Digital Filters. In 11th AIAA/CEAS Aeroacoustics Conference, AIAA 2005-2862, 2005.

[16] R. Ewert, O. Kornow, J.W. Delfs, J. Yin, T. Röber, and M. Rose. A CAA Based Approach to Tone Haystacking. In 15th AIAA/CEAS Aeroacoustics Conference, AIAA 2009-3217, 2009.

[17] R. Ewert and W. Schröder. Acoustic perturbation equations based on flow decomposition via source filtering. Journal of Computational Physics, 188:365-398, 2003.

[18] F. Flemming, A. Sadiki, and J. Janicka. Investigation of combustion noise using a LES/CAA hybrid approach. Proceedings of the Combustion Institute, 31:3189-3196, 2007.

[19] P. Gerlinger. Numerische Verbrennungssimulation - Effiziente Numerische Simulation turbulenter Verbrennung. Springer-Verlag, Germany, 2005. ISBN 3-540-23337-7.

[20] F. Grimm, R. Ewert, J. Dierke, B. Noll, and M. Aigner. Efficient full 3D turbulent combustion noise simulation based on stochastic sound sources. In 21st AIAA/CEAS Aeroacoustics Conference, 2015. AIAA Paper 2015-2973.

[21] C. Hirsch, J. Wäsle, A. Winkler, and T. Sattelmayer. A spectral model for the sound pressure from turbulent premixed combustion. Proceedings of the Combustion Institute, 31(1):14351441, 2007.
[22] F. Hu, M. Hussaini, and J. Manthey. Low- 793 dissipation and low-dispersion runge-kutta 794 schemes for computational acoustics. Journal of 795 Computational Physics, 124(1):177-191, 1996.

[23] M. Ihme, H. Pitsch, and D.J. Bodony. Radiation 797 of noise in turbulent non-premixed flames. Pro- ${ }_{798}$ ceedings of the Combustion Institute, 32:1545- 799 1553, 2009.

[24] F. Joos. Technische Verbrennung: Verbren- 801 nungstechnik, Verbrennungsmodellierung, Emis- 802 sionen. Springer Verlag Berlin Heidelberg, ISBN: $\quad 803$ 978-3-540-34333-2, 2006.

[25] G. Kalitzin, N. Kalitzin, and A. Wilde. A Factorization Scheme for RANS Turbulence Models and SNGR Predictions of Trailing Edge Noise. In 6th AIAA/CEAS Aeroacoustics Conference, 2000. AIAA Paper 2000-1982.

[26] R. Kraichnan. Diffusion by a random velocity field. Physics of Fluids, 13:22-31, 1970.

[27] D.G. Krige. A statistical approach to some basic mine valuation problems on the witwatersrand. $J$. of the Chem., Metal and Mining Soc. of South Africa, 52(6):119-139, 1951.

[28] Y. Liu, A.P. Dowling, N. Swaminathan, R. Morvant, M.A. Macquisten, and L.F. Caracciolo. Prediction of combustion noise for an aeroenginge combustor. Journal of Propulsion and Power, 30:114-122, 2014.

[29] B. Magnussen. On the structure of turbulence and a generalized eddy dissipation concept for chemical reaction in turbulent flow. In 19th AIAA Aerospace Science Meeting, 1981. AIAA 1981-42.

[30] W. Meier, I. Boxx, M. Stöhr, and C.D. Carter. Laser-based investigations in gas turbine model combustors. Experiments of Fluids, 49:865-882, 2010 .

[31] F. Menter and Y. Egorov. The scale-adaptive simulation method for unsteady turbulent flow predictions. part 1: Theory and model description. Flow Turbulence and Combustion, 85(1):113-138, 2010.

[32] F. Menter, M. Kuntz, and R. Langtry. Ten Years of Industrial Experience with the SST Turbulence Model. Turbulence, Heat and Mass Transfer 4, ed: K. Hanjalic, Y. Nagano, and M. Tummers, Begell House, Inc., pages 625-632, 2003.

[33] B. Mühlbauer, R. Ewert, O. Kornow, and B. Noll. Evaluation of the RPM Approach for the Simulation of Broadband Combustion Noise. AIAA Journal, 48(7):1379-1390, 2010. . 
[34] R.M. Munt. Acoustic Transmission Properties of a Jet Pipe with Subsonic Jet Flow: I. The Cold Jet Reflection Coefficient. Journal of Sound and Vibration, 142(3):413-436, 1990.

[35] D.G. Nicol, P.C. Malte, A.J. Hamer, R.J. Roby, and R.C. Steele. Development of a five-step global methane oxidation-no formation mechanism for lean-premixed gas turbine combustion. J. Eng. Gas. Turb. Power, 121(2):272-280, 1999.

[36] O.M. Phillips. On the generation of sound by supersonic turbulent shear layers. J. Fluid Mech., 9:1-28, 1960.

[37] R.J. Purser, W.-S. Wu, D.F. Parrish, and N.M. Roberts. Numerical aspects of the application of recursive filters to variational statistical analysis. part i: Spatially homogeneous and isotropic gaussian covariances. Monthly Weather Review, 131:1524-1535, 2003.

[38] R.J. Purser, W.-S. Wu, D.F. Parrish, and N.M. Roberts. Numerical aspects of the application of recursive filters to variational statistical analysis. part ii: Spatially inhomogeneous and anisotropic gaussian covariances. Monthly Weather Review, 131:1536-1548, 2003.

[39] G. Reichling, B. Noll, and M. Aigner. Development of a Projection-Based Method for the Numerical Calculation of Compressible Reactive Flows. In Proceedings of the 51st AIAA Aerospace Sciences Meeting including the New Horizons Forum and Aerospace Exposition, 2013. AIAA 2013-1003.

[40] G. Reichling, B. Noll, and M. Aigner. Numerical simulation of the non-reactive and reactive flow in a swirled model gas turbine combustor. In 21st AIAA Computational Fluid Dynamics Conference, 2013. AIAA 2013-2434.

[41] C.F. Silva, M. Leyko, F. Nicoud, and S. Moreau. Assessment of combustion noise in a premixed swirled combustor via large-eddy simulation. Computers and Fluids, 78:1-9, 2013.

[42] M. Stöhr, I. Boxx, C.D. Carter, and W. Meier. Experimental study of vortex-flame interaction in a gas turbine model combustor. Combustion and Flame, 159:2636-2649, 2012.

[43] M. Stöhr and S. Werner. Private communication. 2015.

[44] C.K.W. Tam and L. Auriault. Jet Mixing Noise from Fine-Scale Turbulence. AIAA Journal, 37(2):145-153, 1999.

[45] C.K.W. Tam and Z. Dong. Wall boundary conditions for high-order finite-difference schemes in computational aeroacoustics. Theoret. Comput. ${ }^{895}$ Fluid Dynamics, 6:303-322, 1994.

[46] C.K.W. Tam and J.C. Webb. DispersionRelation-Preserving Finite Difference Schemes for Computational Acoustics. Journal of Computational Physics, 107(2):262-281, 1993.

[47] R. Temam. Sur l'approximation de la solution des equations de navier-stokes par la methode des pas fractionnaires. Archive for Rational Mechanics and Analysis, 32:135-153, 1969.

[48] P. Weigand, W. Meier, X.R. Duan, W. Stricker, and M. Aigner. Investigation of swirl flames in a gas turbine model combustor; i. flow field, structures, temperatures and species distributions. Combustion and Flame, 144:205-224, 2006.

[49] F. Zhang, P. Habisreuther, H. Bockhorn, 910 H. Nawroth, and C.O. Paschereit. On Prediction 911 of Combustion Generated Noise with the Tur- 912 bulent Heat Release Rate. Acta Acustica united 9913 with Acustica, 99(6):940-951, 2013.

[50] F. Zhang, P. Habisreuther, M. Hettel, and H. Bockhorn. Numerical Computation of Combustion Induced Noise Using Compressible LES and Hybrid CFD/CAA Methods. Acta Acustica united with Acustica, 98(1):120-134, 2012. 